\title{
NUMERICAL SIMULATIONS AND ASSESSMENTS OF PERFORATED BREAKWATERS
}

\author{
Philip L.-F. Liu, National University of Singapore and Cornell University, philip.liu@nus.edu.sg \\ Pablo Higuera, National University of Singapore, pablo.higuera@nus.edu.sg
}

\section{INTRODUCTION}

In this paper we study the physical processes of regular wave trains impacting on a perforated breakwater. The breakwater consists of an array of vertical rectangular columns and a backwall (see Figure 1). We have performed numerical simulations in which reflection coefficients have been calculated based on the Mansard and Funke (1980) theory and compared with laboratory data and analytical solutions (Kakuno et al. 1992, Kakuno \& Liu, 1993). The numerical solutions will be further analyzed to describe the hydrodynamic processes, identify the limitations of the analytical theory and the scale effects in the laboratory experiments.

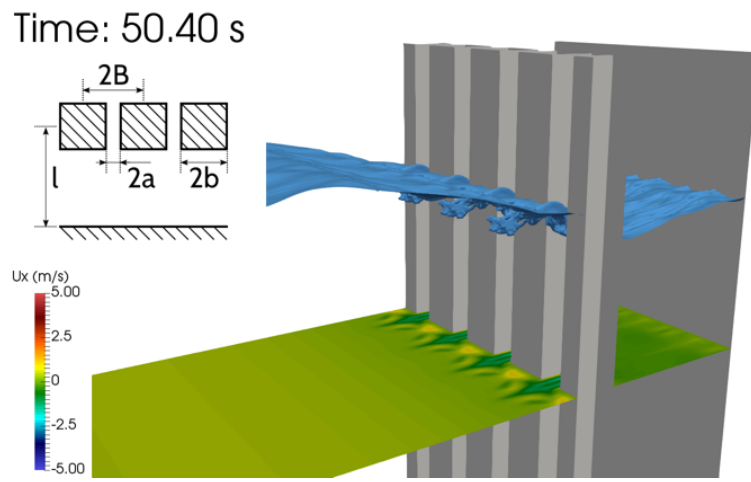

Figure 1 - A 3D snapshot of the free surface near the breakwater chamber for square columns. Horizontal velocity magnitude is represented on a horizontal cutting plane halfway in the water column. A sketch (top left, not to scale) of the dimensions of the system is included.

\section{PREVIOUS STUDIES}

Kakuno et al. (1992) and Kakuno \& Liu (1993) provide an analytical solution for reflection coefficient, based on the potential flow theory and matched asymptotic expansions, for the perforated breakwater configuration sketched in figure 1. The theory matches the near-field and far-field solutions, and introduces a simple energy dissipation model that links the pressure drop across the slit with the average flow velocity in the opening between columns. Only one dimensionless parameter, the energy loss coefficient, $f$, needs to be empirically determined.

As reported in Kakuno et al. (1992), small scale laboratory experiments were performed in a $50 \mathrm{~m}$ long flume, with a still water depth of $\mathrm{h}=0.5 \mathrm{~m}$ and several dimensionless ratios $(a / B, B / l \& h / B)$. The ratio $H / L$ (wave steepness) was kept constant and equal to 0.01 . Regular wave trains were generated and reflection coefficients were measured. Experimental results present an excellent degree of agreement when compared with the analytical solution, using $f=1.5$. One set of such comparisons is shown in Figure 2.

\section{NUMERICAL EXPERIMENTS}

The numerical simulations have been performed with an enhanced version of the 3D Reynolds-Averaged NavierStokes numerical model based on the OpenFOAM library presented in Higuera et al. (2013). The model features an active wave generation and absorption system to avoid the unbounded growth of energy during long simulations. The setup with $\mathrm{a} / \mathrm{B}=0.1525, \mathrm{~B} / \mathrm{l}=$ 0.04966 and $h / B=16.949$ (see Figure 1) has been selected.

The 3D numerical mesh (1.3 million cells) is structured and covers approximately one wavelength in front of the columns. Taking advantage of the lateral symmetry planes, only half of the domain is simulated in the spanwise direction (i.e. half a column and half an opening). The maximum resolution is $5 \mathrm{~mm}$ in the wave propagation direction, $2 \mathrm{~mm}$ in the vertical direction and $0.5 \mathrm{~mm}$ in the spanwise direction, at laboratory scale. A mesh at prototype scale has also been created, multiplying the previous dimensions by a factor of 50 . According to Froude scaling, the time scale factor of the prototype scales simulations is 7 .

Seven cases with $\mathrm{I} / \mathrm{L}$ varying from 0.10 to 0.40 have been modeled with the $k-\omega$ SST turbulence model presented in Devolder et al. (2017). Each of the cases has been simulated for 20-40 wave periods, until the quasi-steady state has been reached. Computations took 20 to 90 hours in parallel (12 Intel Xeon cores, 2.60 $\mathrm{GHz}$ ).

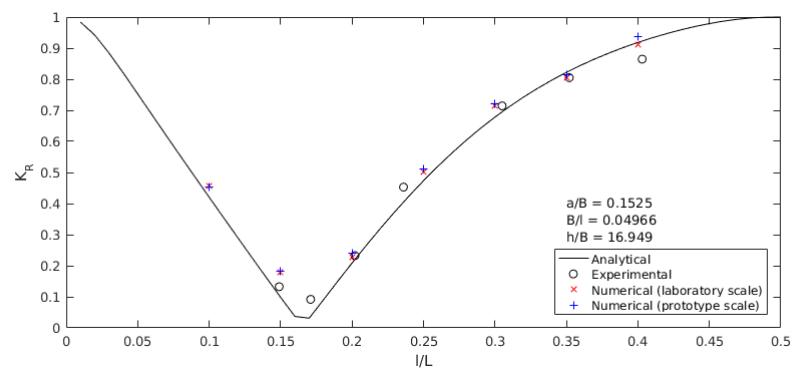

Figure 2 - Reflection coefficient versus dimensionless chamber length $(\mathrm{I} / \mathrm{L})$.

\section{PRELIMINARY RESULTS}

The reflection coefficient analysis as a function of the relative length of the chamber is shown in Figure 2. Results demonstrate an excellent agreement with the first order analytical solution and laboratory experiments presented in Kakuno et al. (1992), both at laboratory scale and prototype scale (LS and PS, from now on).

The theory in Kakuno et al. (1992) and Kakuno \& Liu (1993) was developed in a dimensionless form, therefore, it should be free from scale effects. However, the $f$ 
coefficient introduced to model energy loss may depend on the scale of the problem. The preliminary numerical simulations at PS (+ marker in Figure 2) indicate the absence of scale effects because of the close fit to the theory and numerical results at LS. Nevertheless, the Reynolds number is significantly higher at PS, as it scales with a factor of 350 , namely being approximately 22,500 for LS and 8 million for PS. This means that a much finer mesh would be needed in PS to capture the same relative turbulence scales as in LS. Such analysis will be performed in the future.

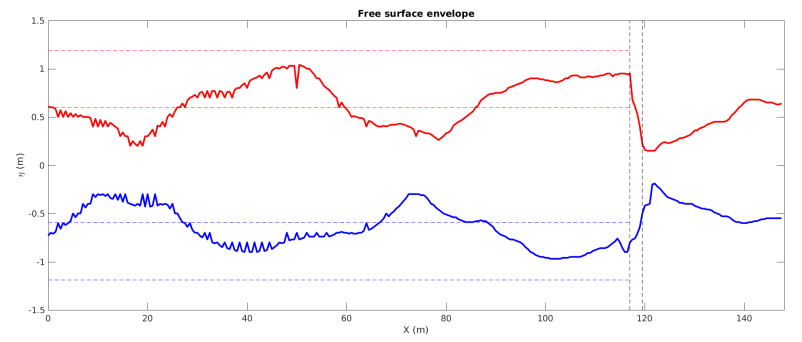

Figure 3 - Envelope of free surface elevation along the PS wave flume for a wave period. Case $\mathrm{I} / \mathrm{L}=0.25$. The cylinder location is indicated with vertical dashes lines.

Figure 3 shows the maximum and minimum free surface elevations along the wave flume during the last wave period simulated for the case of $\mathrm{I} / \mathrm{L}=0.25$. In this particular case the physical conditions originate a node at the cylinders. The horizontal dashed lines mark the bounds for $\pm \mathrm{H} / 2$ and $\pm \mathrm{H}$, which represents the upper limit for full reflection of a linear theory wave. The node and anti-node system can be easily identified outside the chamber. Since the reflection coefficient of this case is $51 \%$, at the nodes the envelope gap is not zero and at the anti-nodes envelope elevations do not reach the $\pm \mathrm{H}$ limits. Inside the chamber, the minimum envelope gap takes place near the row of cylinders, while the maximum envelope gap appears at the fully-reflecting back wall. The nonlinear processes inside the chamber produce energy transfer to higher harmonics and trigger high order resonant frequencies which can be observed in a free surface elevation video.

The difference in elevation between the inside and outside of the chamber induced by the cylinders is significant. A gradient larger than $\mathrm{H} / 2$ appears through the cylinder row for both the upper and lower envelopes. This indicates that during the maximum free surface elevation phase outside the chamber, the water flows into the chamber, but the wave period is not long enough to allow equaling the levels at both sides. The opposite situation occurs for the lowest free surface elevation.

Figure 4 presents the time evolution of the instantaneous potential and kinetic energies of the water volume inside the chamber at laboratory scale. The potential energy achieves a quasi-steady state very fast, after just 8 wave periods. The variation of kinetic energy presents a gradual evolution with an increasing trend, as shown in the moving average line. The growth does not continue after $\mathrm{t}=30 \mathrm{~T}$, when the quasi-steady state is achieved.
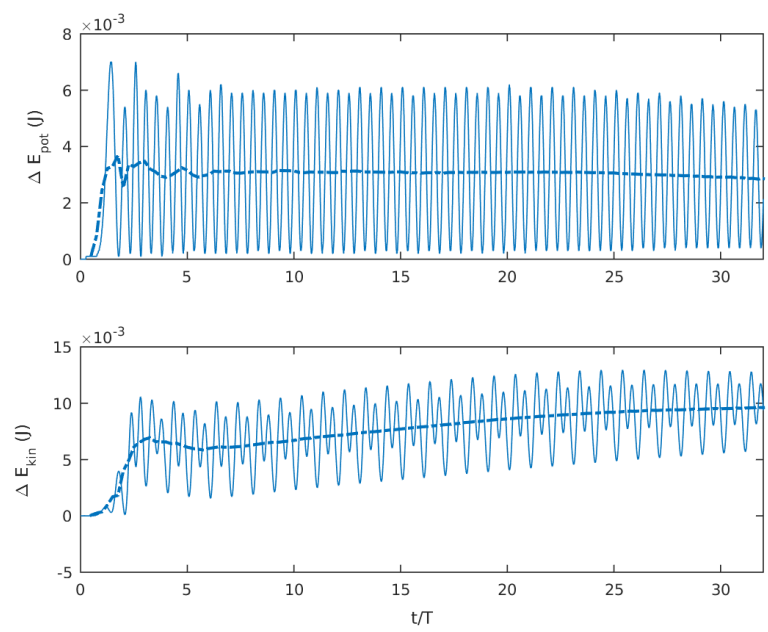

Figure 4 - Evolution of the potential and kinetic energy (top and bottom panels, respectively) inside the chamber. Case $\mathrm{I} / \mathrm{L}=0.25$ at laboratory scale. The dashed line is the moving average over one wave period.

\section{CONCLUSIONS}

The CFD simulations produce results that match well with the theory and experiments in Kakuno et al. (1992). Numerical modelling, although costlier, has proven adequate to study the nonlinear physical processes involved in slit breakwaters. Future work, which will be reported in the presentation, will include a detailed analysis on scale effects.

\section{ACKNOWLEDGEMENTS}

The computational work for this article was partially performed on resources of the National Supercomputing Centre, Singapore (www.nscc.sg).

\section{REFERENCES}

Devolder, Rauwoens \& Troch (2017): Application of a buoyancy-modified k-omega SST turbulence model to simulate wave run-up around a monopile subjected to regular waves using OpenFOAM. Coastal Engineering, vol. 125, pp. 81-94.

Higuera, Lara \& Losada (2013): Realistic wave generation and active wave absorption for Navier-Stokes models: Application to OpenFOAM. Coastal Engineering, vol. 71, pp. 102-118.

Mansard \& Funke (1980): The measurement of incident and reflected spectra using least squares method. Proc. the 17th International Conference on Coastal Engineering, pp. 154-172.

Kakuno, Oda \& Liu (1992): Scattering of waves by vertical cylinders with a backwall. Proceedings of the 23rd International Conference on Coastal Engineering, pp. 1258-1271.

Kakuno \& Liu (1993): Scattering of water waves by vertical cylinders. Journal of Waterway, Port, Coastal and Ocean Engineering, vol. 119, pp. 302-322. 